\title{
Cyclic AMP Signaling Promotes the Differentiation of Human Induced Pluripotent Stem Cells into Intestinal Epithelial Cells ${ }^{\mathbb{\$}}$
}

\author{
Tomoki Kabeya, Shimeng Qiu, Momona Hibino, Mizuka Nagasaki, Nao Kodama, Takahiro Iwao, \\ and Tamihide Matsunaga
}

\begin{abstract}
Department of Clinical Pharmacy, Graduate School of Pharmaceutical Sciences (T.K., S.Q., N.K., T.I., T.M.) and Educational Research Center for Clinical Pharmacy, Faculty of Pharmaceutical Sciences (M.H., M.N., T.I., T.M.), Nagoya City University, Nagoya, Japan
\end{abstract}

Received April 22, 2018; accepted July 25, 2018

\begin{abstract}
To develop a novel in vitro system for predicting intestinal drug absorption using human induced pluripotent stem (iPS) cell-derived intestinal epithelial cells, the cells need to have sufficient drugmetabolizing enzyme and drug transporter activities. We found that cyclic adenosine monophosphate (cAMP) signaling plays an important role in the differentiation of human iPS cells into intestinal epithelial cells. In this study, we aimed to demonstrate the effects of signaling activation in the intestinal differentiation of human iPS cells and the pharmacokinetic characteristics of human iPS cell-derived intestinal epithelial cells. Human iPS cells were differentiated into intestinal stem cells using activin $A$ and fibroblast growth factor 2 . Subsequently, the intestinal stem cells were maturated into intestinal epithelial cells by treatment with 8-bromo-cyclic adenosine monophosphate (8-Br-cAMP) and 3-isobutyl-1-methylxanthine (IBMX),
\end{abstract}

which activate cAMP signaling. The expression levels of intestinal markers and pharmacokinetics-related genes in the differentiated cells were markedly increased by using 8-Br-cAMP and IBMX. In the cells differentiated with the compound we observed cytochrome P450 (CYP) 3A4 inducibility via pregnane $X$ receptor and vitamin D receptor. The metabolic activities of CYP2C9, CYP2C19, CYP2D6, CYP3A4/5, and UDP-glucuronosyltransferase, which are expressed in the human small intestine, were also markedly increased. Furthermore, uptake of glycylsarcosine via peptide transporter 1 was markedly increased. The cells differentiated with the compounds also had drug transporter activities via organic anion transporters and $P$-glycoprotein. This study is the first to report that the activation of cAMP signaling promotes differentiation of human iPS cell-derived intestinal epithelial cells.

\section{Introduction}

In drug disposition, the small intestine is involved not only in the absorption of orally administered drugs but also in their metabolism by cytochrome P450 3A enzyme (Paine et al., 2006; Yoshida et al., 2013; Xie et al., 2016). Therefore, the small intestine and liver are key organs related to the first-pass effect of drugs. Accurately predicting intestinal drug absorption and metabolism is very important in nonclinical studies. Human colon carcinoma cell line Caco-2 is commonly used to predict drug membrane permeability in the small intestine (Hidalgo et al., 1989; Yee, 1997). However, Caco-2 cells differ from normal human small intestine cells in the expression patterns of drug-metabolizing enzymes

This work was supported, in part, by a Grant-in-Aid from the Japan Agency for Medical Research and Development [17937834], from the Japan Society for the Promotion of Science [26293036, 17K08421, 16K15164, 40006308], and by a grant of Takeda Science Foundation and Daiko Foundation.

https://doi.org/10.1124/dmd.118.082123.

S This article has supplemental material available at dmd.aspetjournals.org. and drug transporters (Lennernäs et al., 1996; Schmiedlin-Ren et al., 1997; Nakamura et al., 2002; Sun et al., 2002). Using primary human intestinal epithelial cells for the evaluation of drug membrane permeability would be desirable, but they have poor viability and other characteristics such as short life span that limit their application (Aldhous et al., 2001; Chougule et al., 2012; Takenaka et al., 2014). Therefore, currently, there is no pharmacokinetic system that can simultaneously predict the intestinal metabolism and drug absorption.

To accurately predict the pharmacokinetics of the human small intestine, the development of human induced pluripotent stem (iPS) cell-derived intestinal epithelial cells has been needed, which would have functions similar to normal human intestinal epithelial cells. However, there have been few reports of differentiation of intestinal cells from human iPS cells (Spence et al., 2011; Ogaki et al., 2013, 2015; Iwao et al., 2014, 2015; Ozawa et al., 2015). In addition, the pharmacokinetic characteristics of human iPS-derived intestinal cells have been insufficiently investigated. Spence et al. (2011) reported the production of intestinal organoids, with intestinal morphologic features,

ABBREVIATIONS: 8-Br-cAMP, 8-bromo-cyclic adenosine monophosphate; A-83-01, 3-(6-methyl-2-pyridinyl)- $N$-phenyl-4-(4-quinolinyl)-1H-pyrazole1-carbothioamide; ABC, ATP-binding cassette; BCRP, breast cancer resistance protein; CDX2, caudal type homeobox 2; CES, carboxylesterase; CYP, cytochrome P450 enzyme; DMEM, Dulbecco's modified Eagle's medium; DPP4, dipeptidyl peptidase-4; ER, efflux ratio; FABP2, fatty acid-binding protein 2; FBS, fetal bovine serum; FGF, fibroblast growth factor; HBSS, Hanks' balanced salt solution; HPRT, hypoxanthine phosphoribosyltransferase; IBMX, 3-isobutyl-1-methylxanthine; iPS, induced pluripotent stem; ISX, intestine-specific homeobox; MDR1, multidrug resistance 1; NEAA, minimum essential medium nonessential amino acid solution; OATP, organic anion transporting polypeptide; $P_{\text {app }}$, apparent permeability coefficient; PD98059, 2'-amino-3'-methoxyflavone; PEPT1, peptide transporter 1; P-gp, P-glycoprotein; PSC833, valspodar; PXR, pregnane X receptor; SGLT1, sodium-glucose cotransporter 1; SLC, solute carrier; SULT, sulfotransferase; TEER, transepithelial electrical resistance; UGT, UDP-glucuronosyltransferase. 
TABLE 1

Sequences of primers for real-time polymerase chain reaction analysis

\begin{tabular}{lcc}
\hline \multicolumn{1}{c}{ Gene Name } & Sense $5^{\prime} \rightarrow 3^{\prime}$ & Antisense 5' $\rightarrow 3^{\prime}$ \\
\hline ABCB1/MDR1 & CCCATCATTGCAATAGCAGG & TGTTCAAACTTCTGCTCCTGA \\
ABCG2/BCRP & AGATGGGTTTCCAAGCGTTCAT & CCAGTCCCAGTACGACTGTGACA \\
CDX2 & ACCTGTGCGAGTGGATGC & TCCTTTGCTCTGCGGTTCT \\
CES2A1 & CTGGGGAGTCTTGTCCATGT & GGAAGGGAAGGTCATGTTGA \\
CYP2C9 & GACATGAACAACCCTCAGGACTTT & TGCTTGTCGTCTCTGTCCCA \\
CYP2C19 & GAACACCAAGAATCGATGGACA & TCAGCAGGAGAAGGAGAGCATA \\
CYP3A4 & CTGTGTGTTTCCAAGAGAAGTTAC & TGCATCATCAATTCCTCCTGCAG \\
DPP4 & CAAATTGAAGCAGCCAGACA & GGAGTTGGGAGACCCATGTA \\
FABP2 & TTGGAAGGTAGACCGGAGTG & AGGTCCCCCTGAGTTCAGTT \\
HPRT & CTTTGCTTTCCTTGGTCAGG & TCAAGGGCATATCCTACAACA \\
ISX & CAGGAAGGAAGGAAGAGCAA & TGGGTAGTGGGTAAAGTGGAA \\
PXR & AGGATGGCAGTGTCTGGAAC & AGGGAGATCTGGTCCTCGAT \\
SLC5A1/SGLT1 & CAACATCGCCTATCCAACCT & TAAACAACCTTCCGGCAATC \\
SLC15A1/PEPT1 & CACCTCCTTGAAGAAGATGGCA & GGGAAGACTGGAAGAGTTTTATCG \\
Villin 1 & AGCCAGATCACTGCTGAGGT & TGGACAGGTGTTCCTCCTTC \\
\hline
\end{tabular}

from human pluripotent stem cells using the Matrigel embedding method. However, quantitative evaluation of the intestinal absorption process using three-dimensional intestinal organoids is difficult. Intestinal epithelial cells with monolayers suitable for the quantitative evaluation of pharmacokinetics would be more useful.

Generally, small molecular compounds are useful as inducers of the differentiation of human iPS cells so that they can be stably synthesized in large quantities and their effect are stable. However, to the best of our knowledge, there are few studies on small molecular compounds that can promote the intestinal differentiation of iPS cells. Therefore, the kind of effect useful for differentiation is not well known. We have previously reported that three different inhibitors, transforming growth factor- $\beta$, DNA methyltransferase, and mitogenactivated protein kinase inhibitors, promote the differentiation of human iPS cell-derived intestinal epithelial cells (Iwao et al., 2015; Kodama et al., 2016a). The intestinal epithelial cells differentiated using these inhibitors exhibit activities of drug-metabolizing enzymes, such as cytochrome P450, UDP-glucuronosyltransferase (UGT), sulfotransferase (SULT), and carboxylesterase (CES), and drug transporters such as solute carrier family (SLC) 15 member 1/peptide transporter 1 (SLC15A1/PEPT1) and ATP-binding cassette (ABC) subfamily G member 2/breast cancer resistance protein (ABCG2/BCRP) (Iwao et al., 2015; Kodama et al., 2016b; Kabeya et al., 2017). In addition, we demonstrated that the fraction absorbed of drugs could be predicted from the apparent permeability coefficient $\left(P_{\text {app }}\right)$ in human iPS cell-derived intestinal epithelial cells (Kodama et al., 2016b). However, sufficient transporter activity of P-glycoprotein (P-gp) was not observed; the transporter activity of CYP3A4, a major drug-metabolizing enzyme in the human small intestine, was also low in these cells. To use human iPS cell-derived intestinal epithelial cells for pharmacokinetic studies, it is necessary to improve some of the pharmacokinetic functions. Therefore, we have conducted screening of compounds effective for the intestinal differentiation of human iPS cells.

Recently, we found that 8-bromo-cyclic adenosine monophosphate (8-Br-cAMP) and 3-isobutyl-1-methylxanthine (IBMX), which are small molecular compounds that activate cyclic AMP (cAMP) signaling, promote the differentiation of human iPS cell-derived intestinal epithelial cells. In this study, we demonstrated that cAMP signaling plays an important role in the differentiation of human iPS cells into intestinal epithelial cells. Furthermore, we analyzed the pharmacokinetic functions of the differentiated intestinal epithelial cells.

\section{Materials and Methods}

Materials. Human iPS cell line Windy was provided by Dr. A. Umezawa of the National Center for Child Health and Development (Tokyo, Japan), and another cell line, 610B1, was purchased from Riken BRC (Tsukuba, Japan). Fibroblast growth factor 2 (FGF2), activin A, and epidermal growth factor were purchased from PeproTech (Rocky Hill, NJ). BD Matrigel Matrix Growth Factor Reduced was purchased from BD Biosciences (Bedford, MA). PD98059 (2'-amino-3'-methoxyflavone), 5-aza-2'-deoxycytidine, A-83-01 [3-(6-methyl-2pyridinyl)- $N$-phenyl-4-(4-quinolinyl)- $1 H$-pyrazole-1-carbothioamide], ibuprofen, and sulfobromophthalein were purchased from Wako Pure Chemical Industries (Osaka, Japan). IBMX and PSC833 (valspodar) were purchased from Sigma-Aldrich (St. Louis, MO). Rhodamine 123 was purchased from Dojindo Laboratories (Kumamoto, Japan). 8-Br-cAMP was purchased from Enzo Life Sciences (New York, NY). Anti-villin and anti-PEPT1 antibodies were purchased from Santa Cruz Biotechnology (Dallas, TX). Anti-occludin antibody was purchased from Invitrogen Life Technologies/Thermo Fisher Scientific (Carlsbad, CA). Anti-P-glycoprotein antibody was purchased from Abcam (Cambridge, United Kingdom). Human adult small intestine total RNA (five donors) was purchased from BioChain Institute (Newark, CA). All other reagents were of the highest quality available.

Human iPS Cell Culture. Human iPS cell lines were cultured on a feeder layer using a 1:1 mixture of Dulbecco's modified Eagle's medium (DMEM) and Ham's nutrient mixture F-12 (DMEM/F-12) supplemented with 20\% KnockOut Serum Replacement, $2 \mathrm{mM}$ L-glutamine, $1 \%$ minimum essential medium nonessential amino acid solution (NEAA), $0.1 \mathrm{mM}$ 2-mercaptoethanol, and $5 \mathrm{ng} / \mathrm{ml} \mathrm{FGF2}$. Mitomycin C-treated mouse embryonic fibroblasts were used as feeder cells.

Differentiation of Human iPS Cells into Intestinal Epithelial Cells. Differentiation of the intestinal epithelial cells was performed according to the protocol reported elsewhere (Iwao et al., 2015). Briefly, for definitive endoderm differentiation, human iPS cells were incubated with activin A for 3 days. For the induction of intestinal stem cells, the cells were subsequently incubated with FGF2 for 4 days. Then, the intestinal stem cells were cultured in the intestinal differentiation medium DMEM/F-12 containing 2\% fetal bovine serum (FBS), $2 \%$ B-27 supplement, 1\% N2 supplement, 1\% NEAA, 2 mM L-glutamine, $100 \mathrm{U} / \mathrm{ml}$ penicillin, $100 \mu \mathrm{g} / \mathrm{ml}$ streptomycin, and $20 \mathrm{ng} / \mathrm{ml}$ of epidermal growth factor for 7 days. Subsequently, these cells were cultured for 12 days in the intestinal differentiation medium supplemented with PD98059, 5-aza-2'deoxycitidine, and A-83-01. In addition, $1 \mathrm{mM} 8$-Br-cAMP and $500 \mu \mathrm{M}$ IBMX were also added to the intestinal differentiation medium.

Caco-2 Cell Culture. Caco-2 cells (RCB0988; Riken BRC) were maintained in DMEM supplemented with $10 \%$ FBS, $1 \%$ NEAA, $100 \mathrm{U} / \mathrm{ml}$ penicillin, and $100 \mu \mathrm{g} / \mathrm{ml}$ streptomycin for 14 days.

RNA Isolation and Reverse Transcription Polymerase Chain Reaction. Total RNA isolation and cDNA synthesis of human iPS cell-derived intestinal epithelial cells and Caco-2 cells were performed according to a protocol reported elsewhere (Kabeya et al., 2017). The relative mRNA expression levels were 

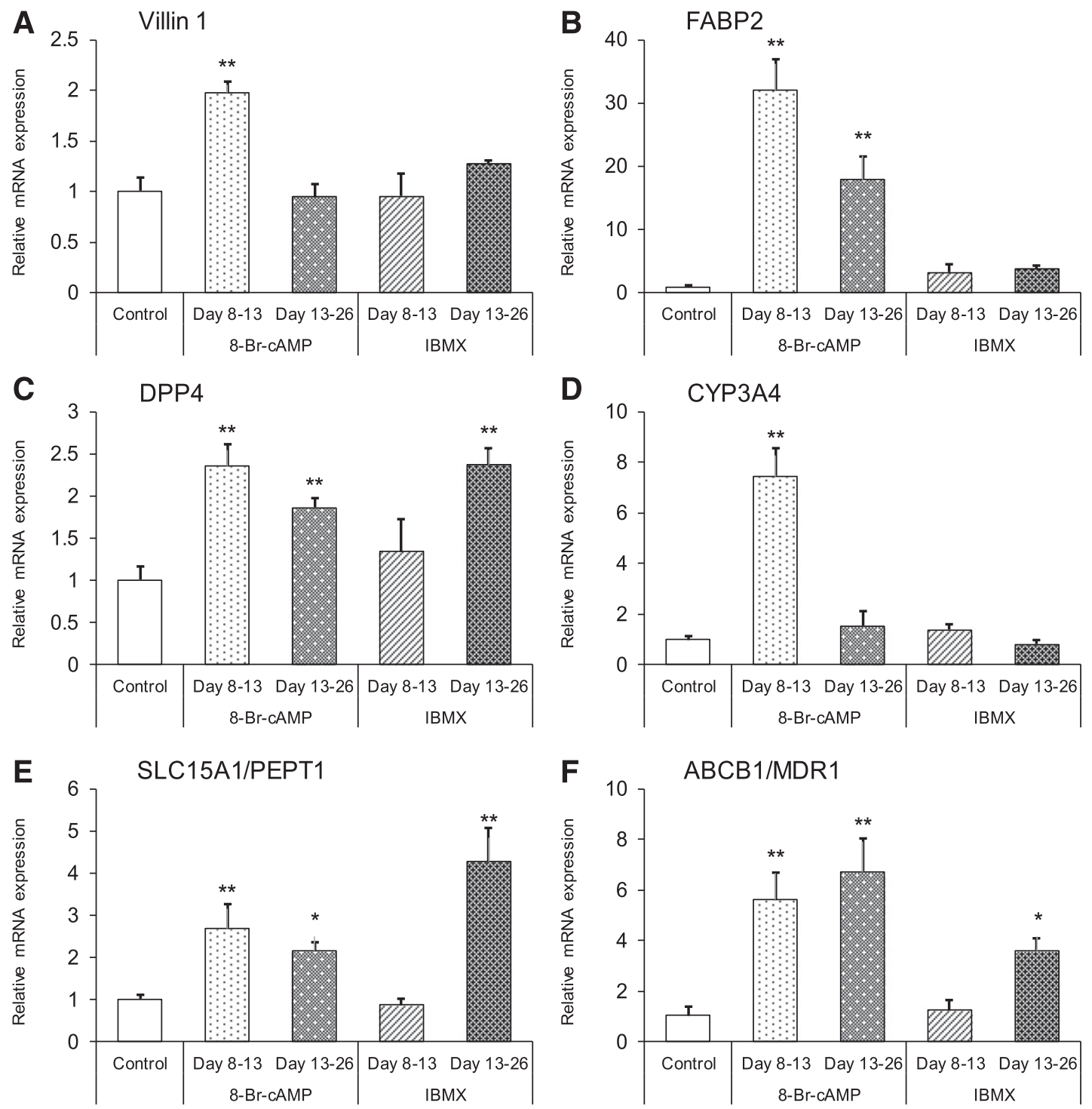

Fig. 1. Effect of 8-Br-cAMP and IBMX on differentiation of human iPS cells into intestinal epithelial cells. Human iPS cells were differentiated into intestinal stem cells by treatment with activin A and FGF2. The intestinal stem cells were then cultured in the intestinal differentiation medium, as described in Materials and Methods. In addition, $1 \mathrm{mM}$ 8-Br-cAMP and $500 \mu \mathrm{M}$ IBMX were added on days 8-13 or 13-26 during differentiation. At terminal differentiation, the total RNA was extracted, and the gene expression levels of intestinal markers $(\mathrm{A}-\mathrm{C})$ and pharmacokinetics-related genes (D-F) were measured by real-time reverse-transcription polymerase chain reaction. HPRT expression levels were used to normalize target gene expression levels. Gene expression levels in the control group were defined as 1 . All data are presented as mean \pm S.D. $(n=3)$. Levels of statistical significance compared with the control group: $* P<0.05 ; * * P<0.01$.

determined using a KAPA SYBR Fast qPCR Kit (Genetics, Tokyo, Japan) and the Applied Biosystems 7300 Real-time Polymerase Chain Reaction System with 7300 System SDS, software version 1.4 (Applied Biosystems, Foster City, CA). The primer pairs are listed in Table 1. Hypoxanthine phosphoribosyltransferase (HPRT) was used as a housekeeping gene for normalizing the mRNA expression levels of target genes.

Determination of Drug-Metabolizing Enzyme Activity. Drug metabolism study was performed according to the protocol previously reported elsewhere (Iwao et al., 2015). At terminal differentiation, the differentiated cells were incubated with intestinal differentiation medium containing substrate cocktail for 24 hours at $37^{\circ} \mathrm{C}$. In the metabolic study of midazolam, the differentiated and Caco- 2 cells were incubated in the presence or absence of $10 \mu \mathrm{M}$ ketoconazole. To normalize drug-metabolizing enzyme activities, the total protein contents of the differentiated and Caco-2 cells were measured using a TaKaRa BCA Protein Assay Kit (Takara Bio, Shiga, Japan), according to the manufacturer's instructions.

Immunofluorescence Staining. For staining of villin and occludin, the differentiated cells were fixed and permeabilized in methanol $\left(-20^{\circ} \mathrm{C}\right)$ for 5 minutes at $4^{\circ} \mathrm{C}$. For staining of PEPT1 and P-gp, the cells were fixed in a $4 \%(w / v)$ paraformaldehyde solution for 30 minutes and permeabilized in a $0.1 \%$ Triton X-100 solution for 5 minutes at room temperature. After washing 3 times with phosphatebuffered saline without calcium and magnesium, the cells were blocked in phosphatebuffered saline containing 5\% FBS for 20 minutes at room temperature. The cells were then incubated for 60 minutes at room temperature with the primary antibodies. The primary antibodies were diluted as follows: villin, 1:100; occludin, 1:100; PEPT1, 1:50; and P-gp, 1:20. The experiments were performed according to the method previously reported elsewhere (Iwao et al., 2015).

Uptake Study. Glycylsarcosine was chosen as a substrate to measure the activity of PEPT1, and $3 \mathrm{mM}$ ibuprofen was used as the specific inhibitor (Omkvist et al., 2010). Before the uptake study, the differentiated cells were preincubated for 20 minutes with a transport buffer [Hanks' balanced salt solution (HBSS) containing $10 \mathrm{mM}$ 4-morpholineethanesulfonic acid, $\mathrm{pH}$ 6.0], with or without the inhibitor. For the uptake study of organic anion transporting polypeptide (OATP), $5 \mathrm{nM}\left[{ }^{3} \mathrm{H}\right]$-estrone-3-sulfate and $200 \mu \mathrm{M}$ sulfobromophthalein were used as the substrate and specific inhibitor, respectively (Sai et al., 2006). HBSS containing $10 \mathrm{mM}$ HEPES (pH 7.4) was used as a transport buffer. The uptake studies were performed according to the previously reported protocol 

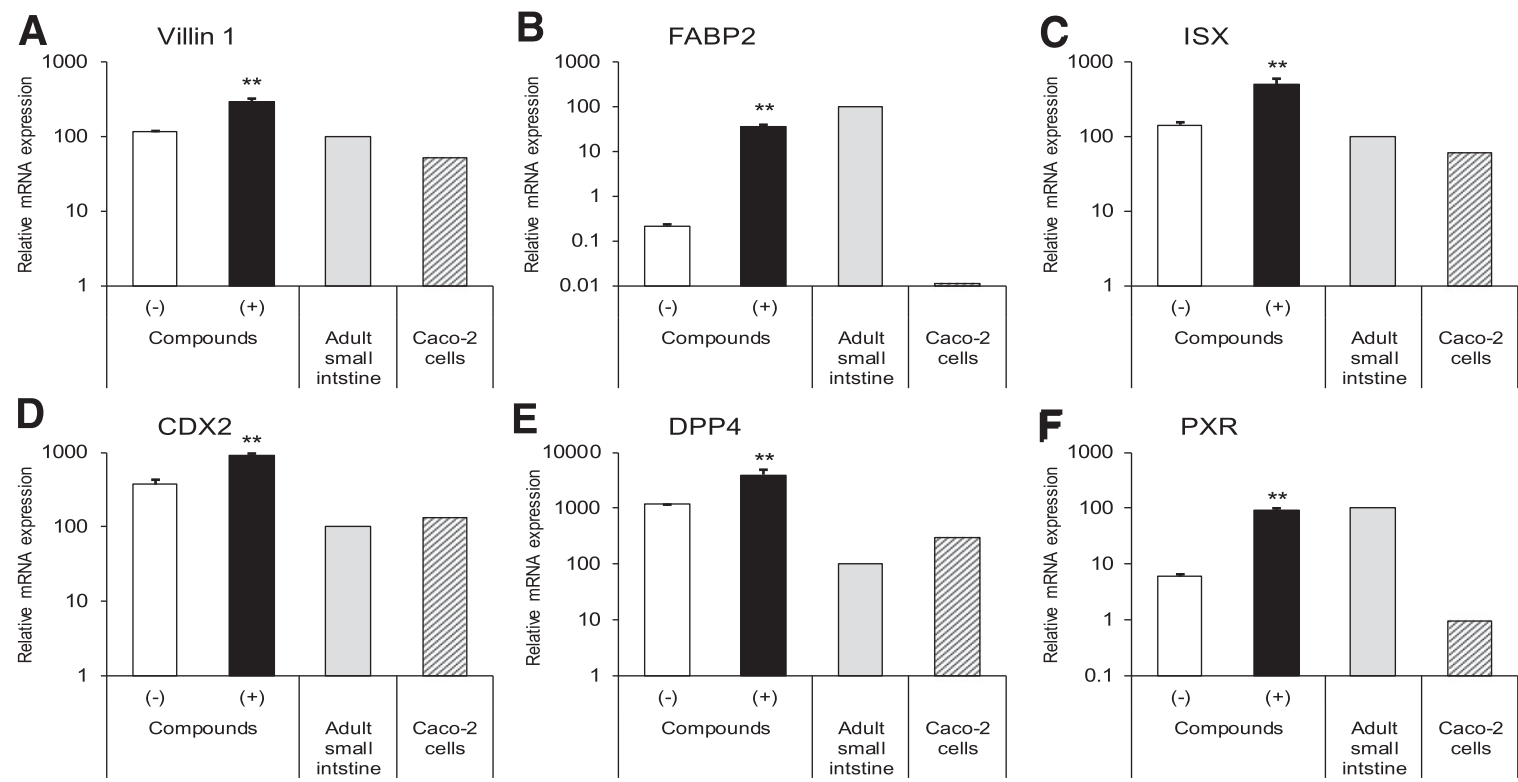

$\mathbf{E}$
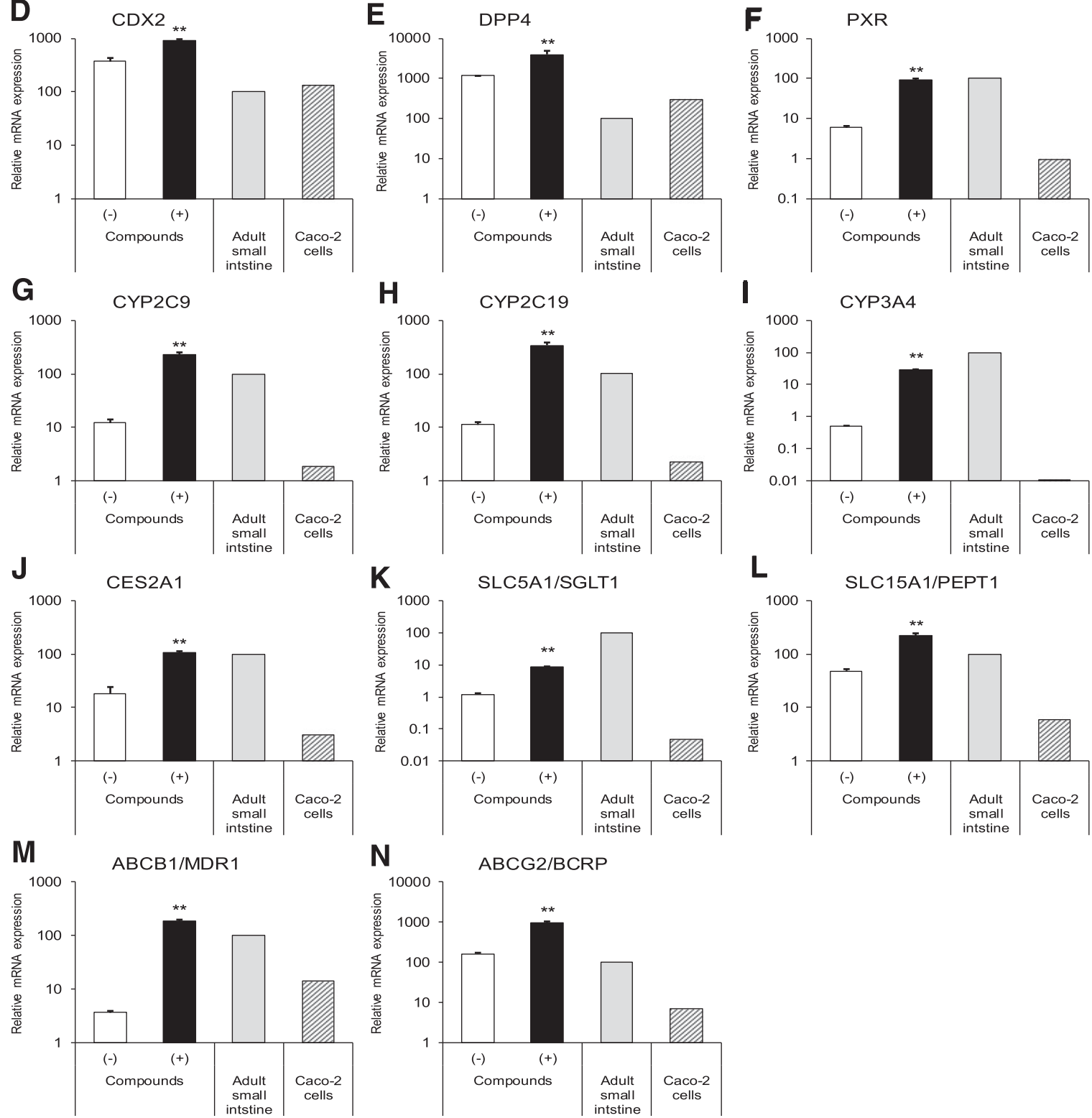

Fig. 2. Relative gene expression levels of intestinal markers (A-E) and pharmacokinetics-related genes (F-N) in differentiated intestinal epithelial cells. During the differentiation, human iPS cell-derived intestinal stem cells were differentiated in the presence or absence of 8-Br-cAMP and IBMX in combination. One millimolar 8-Br-cAMP and $500 \mu \mathrm{M}$ IBMX were added on days 8-14 and 14-26, respectively. At terminal differentiation, the total RNA was extracted, and the gene expression levels of intestinal markers and pharmacokinetics-related genes were measured by real-time reverse-transcription polymerase chain reaction. HPRT expression levels were used to normalize target gene expression levels. Gene expression levels in adult small intestine were defined as 100. All data are presented as mean \pm S.D. $(n=3)$. Level of statistical significance compared with the group without compounds: $* * P<0.01$.

(Iwao et al., 2015). To normalize transporter activities, the total protein contents of the differentiated cells were measured using a TaKaRa BCA Protein Assay Kit, according to the manufacturer's instructions.

Bidirectional Transport Assay. The differentiated cells were seeded on the cell culture inserts. The transepithelial electrical resistance (TEER) values were measured using Millicell ERS-2 (Millipore, Bedford, MA). Rhodamine 123 was chosen as a substrate to measure the activity of P-gp, and $2 \mu \mathrm{M}$ PSC 833 was used as the specific inhibitor (Lee et al., 1994; Jouan et al., 2016). The cells were preincubated for 20 minutes with a transport buffer (HBSS containing $10 \mathrm{mM}$ HEPES, $\mathrm{pH}$ 7.4) in the presence or absence of the inhibitor. 


\section{A Villin/DAPI B PEPT1/DAPI C P-gp/DAPI}

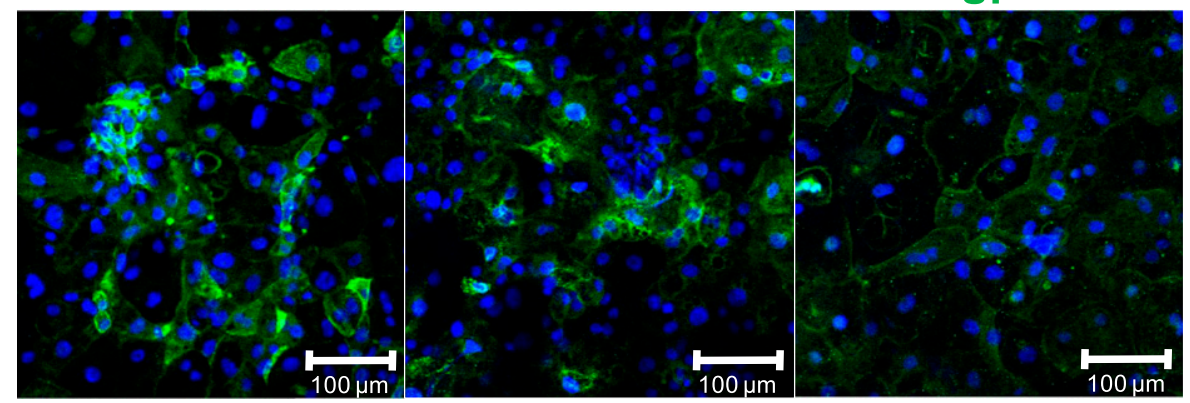

Fig. 3. Immunofluorescence staining of villin (A), PEPT1 (B), and P-gp (C) in the differentiated intestinal epithelial cells by 8 -Br-cAMP and IBMX in combination. After differentiation, the differentiated cells were stained with villin (green), PEPT1 (green), P-gp (green), and 4',6-diamidino-2-phenylindole (DAPI, blue). Scale bar, $100 \mu \mathrm{m}$.

After preincubation, the transport buffer containing $100 \mu \mathrm{M}$ rhodamine 123 was added to the apical or basal chambers, and the cells were incubated at $37^{\circ} \mathrm{C}$ for 120 minutes in the presence or absence of the inhibitor. The samples were collected from the receiver chambers. The concentration of rhodamine 123 was quantified using Synergy HTX (BioTek, Winooski, VT). The excitation and emission wavelengths of rhodamine 123 were 485 and $535 \mathrm{~nm}$, respectively.

Analysis of Apparent Membrane Permeability. The $P_{\text {app }}$ value and efflux ratio (ER) of rhodamine 123 in bidirectional transport assay were calculated according to the analytical method reported elsewhere (Kodama et al., 2016b):

$$
P_{a p p}=\frac{d Q}{d t} \times \frac{1}{A \times C_{0}}
$$

where $\mathrm{d} Q / \mathrm{d} t$ is the amount of the compound permeated per unit of time, $A$ is the membrane surface area, and $C_{0}$ is the initial donor concentration of the compound. The ER of rhodamine 123 was calculated according following equation:

$$
\mathrm{ER}=\frac{P_{\text {app }, \text { BtoA }}}{P_{\text {app }, \text { AtoB }}}
$$

Where $P_{\text {app,AtoB }}$ and $P_{\text {app,BtoA }}$ are $P_{\text {app }}$ values in apical-to-basal and basalto-apical transport.

\section{Statistical Analysis}

The level of statistical significance was assessed using Student's $t$ test for comparisons between two groups or analysis of variance followed by Dunnett's test for multiple comparisons.

\section{Results}

Effect of cAMP Signaling on Differentiation from Human iPS Cells into Intestinal Epithelial Cells. Human iPS cell-derived intestinal stem cells were treated with $1 \mathrm{mM} 8$-Br-cAMP or $500 \mu \mathrm{M}$ IBMX on days 8 to 13 or days 13 to 26 during the intestinal differentiation. After terminal differentiation, the expression levels of intestinal specific markers, such as villin 1 (Boller et al., 1988; Landry et al., 1994), fatty acidbinding protein 2 (FABP2) (Gajda and Storch, 2015), dipeptidyl peptidase4 (DPP4) (Darmoul et al., 1994), and pharmacokinetics-related genes such as CYP3A4 (Paine et al., 2006; Xie et al., 2016), SLC15A1/PEPT1, and ABCB1/multidrug resistance 1 (MDR1) (Hilgendorf et al., 2007; Yoshida et al., 2013), were markedly increased in the cells treated with 8-Br-cAMP and IBMX compared with the cells differentiated without these compounds (control group) (Fig. 1).

Based on these results, 8-Br-cAMP and IBMX were added on days 8 to 14 and 14 to 26 , respectively. In the cells differentiated with these two compounds, the expression levels of villin 1, FABP2, intestine-specific homeobox (ISX) (Choi et al., 2006), caudal type homeobox 2 (CDX2) (Suh and Traber, 1996; Coskun et al., 2011), DPP4, pregnane X receptor (PXR), CYP2C9, CYP2C19, CYP3A4, CES2A1, SLC15A1/PEPT1, SLC5A1/sodium-glucose cotransporter 1 (SGLT1) (Wright and Turk, 2004), $\mathrm{ABCB} 1 / \mathrm{MDR} 1$, and ABCG2/BCRP were markedly increased compared with the cells differentiated without the compounds (Fig. 2). The expression of the proteins villin, PEPT1, and P-gp was also increased (Fig. 3).

CYP3A4 Inducibility and Drug-Metabolizing Enzyme Activities. Before differentiation, $1 \alpha, 25$-dihydroxyvitamin $\mathrm{D}_{3}$ or rifampicin, inducers of CYP3A4, was administered for 48 hours. In CYP3A4, the mRNA

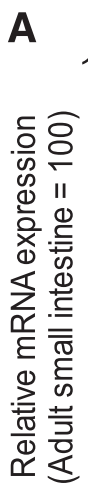
0.01 iPS cell-derived intestinal epithelial cells

B

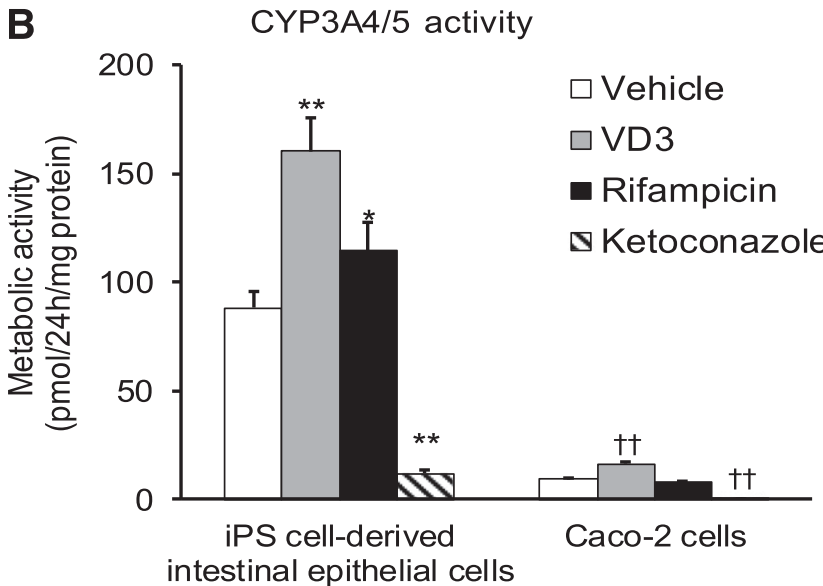

Fig. 4. Induction of CYP3A4 mRNA expression levels (A) and CYP3A4/5 activity (B) in differentiated intestinal epithelial cells by $8-\mathrm{Br}$-cAMP and IBMX in combination. Differentiated cells and Caco-2 cells were treated with $10 \mathrm{nM} 1 \alpha, 25$-dihydroxyvitamin $\mathrm{D}_{3}$ (VD3) or $40 \mu \mathrm{M}$ rifampicin for 48 hours at terminal differentiation. For analysis of CYP3A4 mRNA expression, the total RNA was extracted, and the gene expression levels were measured by real-time reverse-transcription polymerase chain reaction after differentiation. HPRT expression levels were used to normalize target gene expression levels. For analysis of CYP3A4/5 activity at terminal differentiation, the differentiated cells were incubated with intestinal differentiation medium containing $5 \mu \mathrm{M}$ midazolam for 24 hours at $37^{\circ} \mathrm{C}$ in the presence or absence of $10 \mu \mathrm{M}$ ketoconazole. The supernatants were collected, and the metabolites were measured by ultraperformance liquid chromatography with tandem mass spectrometry. Gene expression levels in adult small intestine were defined as 100. All data are presented as mean \pm S.D. $(n=3-5)$. Levels of statistical significance: $* P<0.05 ; * * P<0.01$ compared with the vehicle group in iPS cell-derived intestinal epithelial cells. ${ }^{\dagger \dagger} P<0.01$ compared with the vehicle group in Caco- 2 cells. 
TABLE 2

Drug-metabolizing enzyme activities of CYP1A, CYP2B6, CYP2C9, CYP2C19, CYP2D6, CYP3A4/5, UGT, and SULT in the differentiated intestinal epithelial cells

At terminal differentiation, the differentiated cells were incubated with intestinal differentiation medium containing $40 \mu \mathrm{M}$ phenacetin, $50 \mu \mathrm{M}$ bupropion, $5 \mu \mathrm{M}$ diclofenac, $47.5 \mu \mathrm{M}$ (S)-mephenytoin, $5 \mu \mathrm{M}$ bufuralol, $5 \mu \mathrm{M}$ midazolam, and $10 \mu \mathrm{M}$ 7-hydroxycoumarin for 24 hours at $37^{\circ} \mathrm{C}$. The supernatants were collected, and the metabolites in the differentiated cells with or without 8-Br-cAMP and IBMX in combination were measured by ultraperformance liquid chromatography with tandem mass spectrometry. All data are presented as mean \pm S.D. $(n=4)$.

\begin{tabular}{|c|c|c|c|c|c|}
\hline \multirow[t]{2}{*}{ Enzymes } & \multirow[t]{2}{*}{ Substrate } & \multirow[t]{2}{*}{ Metabolite } & \multicolumn{2}{|c|}{$\begin{array}{c}\text { Metabolic activities } \\
\text { (pmol/24 hour per milligram protein) }\end{array}$} & \multirow[t]{2}{*}{ Ratio (+/-) } \\
\hline & & & Compounds (-) & Compounds (+) & \\
\hline CYP1A & Phenacetin & Acetaminophen & $81.5 \pm 8.4$ & $45.8 \pm 7.6^{a}$ & 0.56 \\
\hline CYP2B6 & Bupropion & Hydroxybupropion & $6.8 \pm 0.9$ & $4.0 \pm 0.8^{a}$ & 0.59 \\
\hline CYP2C9 & Diclofenac & $4^{\prime}$-Hydroxydiclofenac & $0.2 \pm 0.2$ & $15.5 \pm 3.4^{a}$ & 77.5 \\
\hline CYP2C19 & (s)-Mephenytoin & 4'-Hydroxymephenytoin & $0.6 \pm 0.1$ & $28.6 \pm 4.4^{a}$ & 47.7 \\
\hline CYP2D6 & Bufuralol & 1'-Hydroxybufuralol & $0.5 \pm 0.1$ & $0.8 \pm 0.1^{a}$ & 1.60 \\
\hline CYP3A $4 / 5$ & Midazolam & 1'-Hydroxymidazolam & $23.7 \pm 2.6$ & $88.2 \pm 7.9^{a}$ & 3.72 \\
\hline UGT & 7-Hydroxycoumarin & 7-Hydroxycoumarin glucuronide & $2499 \pm 220$ & $4696 \pm 539^{a}$ & 1.88 \\
\hline SULT & & 7-Hydroxycoumarin sulfate & $9949 \pm 3061$ & $5744 \pm 909^{b}$ & 0.58 \\
\hline
\end{tabular}

${ }^{a} \mathrm{P}<0.01$, compared with the group without compounds.

${ }^{b} \mathrm{P}<0.05$, compared with the group without compounds.

expression level and CYP3A4/5 metabolic activity were significantly induced by these ligands in cells differentiated with 8-Br-cAMP and IBMX. CYP3A4/5 activity was increased by 1.8 - or 1.3 -fold by treatment with $1 \alpha, 25$-dihydroxyvitamin $\mathrm{D}_{3}$ or rifampicin, respectively, compared with the vehicle group. The activity was strongly inhibited by ketoconazole, a CYP3A4-specific inhibitor. On the other hand, the mRNA expression level and metabolic activity were significantly increased only by $1 \alpha, 25$-dihydroxyvitamin $\mathrm{D}_{3}$ in Caco2 cells. CYP3A $4 / 5$ activity in Caco- 2 cells was increased by 1.8 -fold by treatment with $1 \alpha, 25$-dihydroxyvitamin $\mathrm{D}_{3}$ (Fig. 4). In cells differentiated with 8-Br-cAMP and IBMX, the metabolic activities of CYP2C9, CYP2C19, CYP2D6, CYP3A4/5, and UGT were markedly increased by 78-, 48-, 1.6-, 3.7-, and 1.9-fold, respectively, compared with cells differentiated without these compounds (Table 2).

Uptake Transport Activities via PEPT1 and OATP. The activity of PEPT1 was measured using glycylsarcosine, a PEPT1 substrate. The activity of PEPT1 in the cells differentiated with 8-Br-cAMP and IBMX was $8.1 \mathrm{pmol} / \mathrm{h}$ per milligram protein, and it was markedly increased 2.3fold compared with the nontreatment group. The activities were dramatically suppressed by treatment with ibuprofen, a PEPT1 inhibitor, and at $4^{\circ} \mathrm{C}$ (Fig. 5A). The rate of uptake of estrone-3-sulfate, an OATP substrate, was $0.28 \mathrm{pmol} / \mathrm{h}$ per milligram protein. The activity was markedly inhibited by sulfobromophthalein, an OATP inhibitor (Fig. 5B).

Efflux Transport Activity via P-gp. On day 7 after differentiation, the cells were seeded on cell culture inserts and treated with 8-Br-cAMP and IBMX. The TEER values of the cells were increased in a timedependent manner and finally reached 200-250 $\Omega \cdot \mathrm{cm}^{2}$ (Fig. 6A). Occludin, a tight junction marker (Groschwitz and Hogan, 2009), was expressed in the cells and localized along the plasma membrane (Fig. $6 \mathrm{~B})$. In the efflux transport study via $\mathrm{P}$-gp using rhodamine 123 as the substrate, the apical-to-basal $P_{\text {app }}$ values were $0.54 \pm 0.31$ and $1.47 \pm$ $0.22\left(\times 10^{-6} \mathrm{~cm} / \mathrm{s}\right)$ in the absence and presence of PSC833, a specific P-gp inhibitor, respectively. On the other hand, the basal-to-apical $P_{\text {app }}$ values were $1.87 \pm 0.34$ and $1.66 \pm 0.14\left(\times 10^{-6} \mathrm{~cm} / \mathrm{s}\right)$ in the absence and presence of PSC833, respectively. The ER value, calculated from the apical-to-basal and basal-to-apical $P_{\text {app }}$ values, was decreased from 3.5 to 1.1 by PSC833 (Fig. 6C)

\section{Discussion}

It has been reported that cAMP signaling promotes the differentiation of pluripotent stem cells into hepatocytes, pancreatic $\beta$ cells, and vascular endothelial cells (Ogawa et al., 2013; Sakano et al., 2014; Ikuno et al., 2017). However, there is no such report on the differentiation of intestinal epithelial cells. The present study initially revealed that the
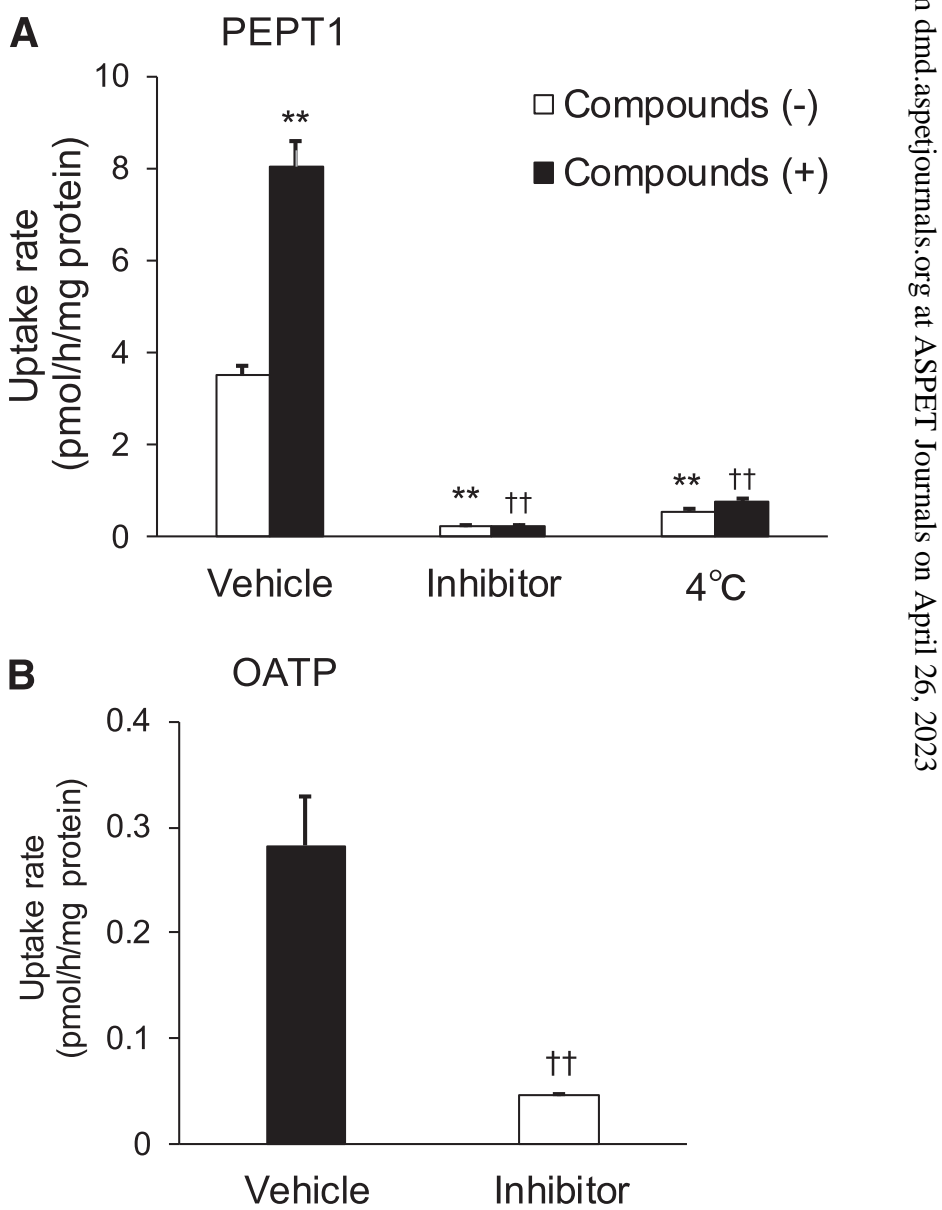

Fig. 5. Uptake transporter activities of PEPT1 (A) and OATP (B) in differentiated intestinal epithelial cells. At terminal differentiation, the cells were incubated with the transport buffer containing glycylsarcosine or estrone-3-sulfate at $37^{\circ} \mathrm{C}$ in the presence or absence of the inhibitors, or at $4^{\circ} \mathrm{C}$ for 60 minutes. The uptake rates of these substrates in the differentiated cells with or without 8-Br-cAMP and IBMX in combination were measured. All data are presented as mean \pm S.D. $(n=3)$. Levels of statistical significance: $* * P<0.01$ compared with the vehicle group without compounds, and ${ }^{\dagger \dagger} P<0.01$ compared with the vehicle group with compounds. 
A

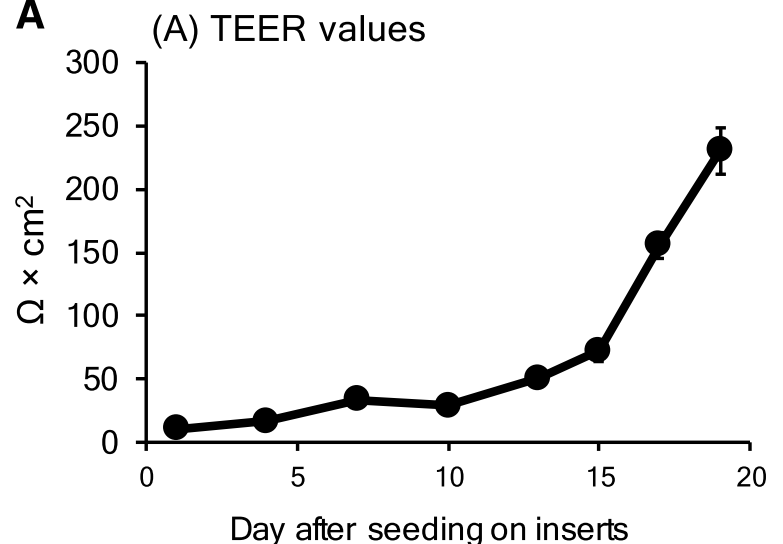

Day after seeding on inserts

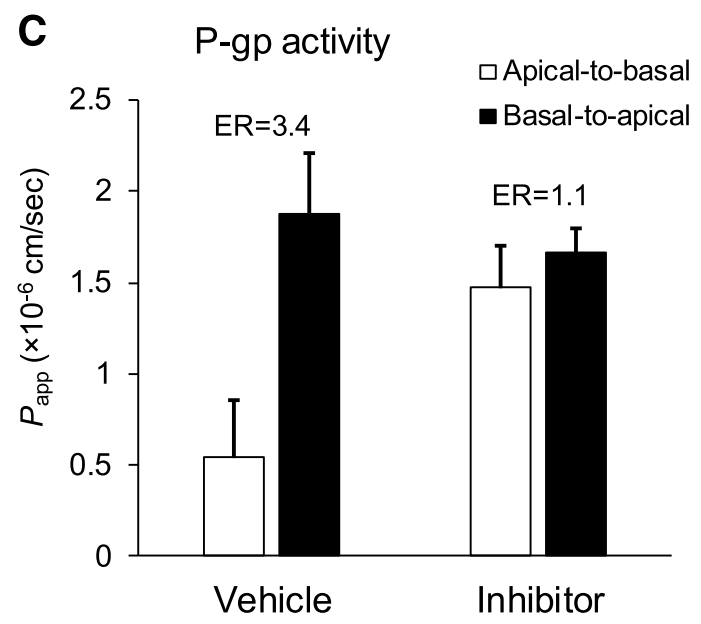

\section{B Villin/Occludin}

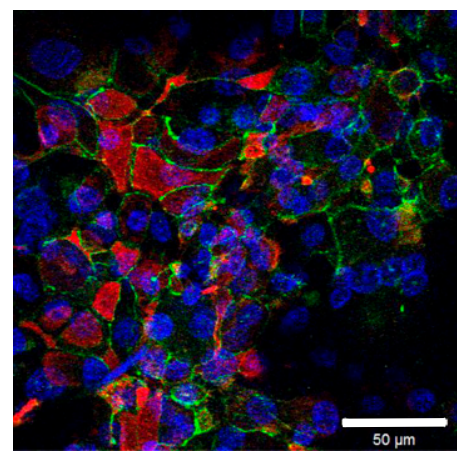

Fig. 6. TEER values, protein expression, and bidirectional permeability of rhodamine 123 in differentiated intestinal epithelial cells by 8-Br-cAMP and IBMX in combination. (A) The TEER values of the cells seeded on culture inserts were measured during intestinal differentiation. (B) The differentiated cells were stained with villin (red), occludin (green), and 4',6-diamidino-2-phenylindole (DAPI, blue) at terminal differentiation. Scale bar, $50 \mu \mathrm{m}$. (C) The cells were incubated with the transport buffer containing rhodamine 123 at $37^{\circ} \mathrm{C}$ for 120 minutes in the presence or absence of the inhibitor. activation of cAMP signaling contributes to the differentiation and maturation of human iPS cell-derived intestinal epithelial cells.

The activation of cAMP signaling in intestinal cell lines has been reported to increase the expression of $\mathrm{CDX} 2$, which is a transcription factor involved in the differentiation into the intestine (Suh and Traber, 1996; Chen et al., 2005; Coskun et al., 2011). Indeed, the expression levels of intestinal markers, including CDX2 and pharmacokinetics-related genes, in human iPS cell-derived intestinal epithelial cells were markedly increased by using 8-Br-cAMP and IBMX during differentiation in our study (Fig. 2). According to immunostaining, the expression of intestinal markers villin, PEPT1, and P-gp was also observed in cells differentiated by a combination of 8-Br-cAMP and IBMX (Fig. 3). Furthermore, when another human iPS cell line, 610B1, was differentiated into intestinal epithelial cells by using 8-Br-cAMP and IBMX, the expression levels of intestinal markers and pharmacokinetics-related genes as well as those of the human iPS cell line Windy were increased (Supplemental Fig. 1). These results suggest that the activation of cAMP signaling is useful for the promotion of differentiation into intestinal epithelial cells against multiple human iPS cell lines.

The induction of CYP3A4 via nuclear receptors, such as PXR, vitamin D receptor, and glucocorticoid receptor, in human iPS cellderived intestinal epithelial cells has been reported (Negoro et al., 2016). In our study, the CYP3A4 mRNA expression level and CYP3A4/5 activity were increased by $1 \alpha, 25$-dihydroxyvitamin $\mathrm{D}_{3}$ and rifampicin in cells differentiated by a combination of 8-Br-cAMP and IBMX (Fig. 4). On the other hand, in Caco-2 cells, a marked increase was observed with treatment with $1 \alpha, 25$-dihydroxyvitamin $\mathrm{D}_{3}$, but not with rifampicin. The CYP3A4/5 activity in human iPS cell-derived intestinal epithelial cells was much higher than that in Caco-2 cells (Fig. 4). These results indicate that human iPS cell-derived intestinal epithelial cells would be a more useful model than Caco- 2 cells for studies of CYP3A4 activity and induction mediated by nuclear receptors, including PXR.

The human small intestine expresses multiple CYP enzymes and metabolizes various clinical drugs (Paine et al., 2006; Xie et al., 2016). The metabolic activities of CYP2C9, CYP2C19, CYP2D6, CYP3A4/5, and UGT, which are expressed in the human small intestine, were markedly increased by using 8-Br-cAMP and IBMX (Table 2). These results suggest that the activation of cAMP signaling contributes to the enhancement of metabolic functions of human iPS cell-derived intestinal epithelial cells. On the other hand, the drug metabolic activities of CYP1A and CYP2B6, which are expressed in nonintestinal tissues, were markedly decreased (Table 2).

From these results, we believe the activation of cAMP signaling in human iPS cell-derived intestinal cells to more specifically promote differentiation into intestinal epithelial cells but not other tissue cells. However, the drug-metabolizing enzyme activities in the differentiated cells are still not high enough. To use human iPS cell-derived intestinal epithelial cells for pharmacokinetic studies, it would be necessary to use cell lines that are more suitable for intestinal differentiation and to improve the protocol.

The peptide transporter SLC15A1/PEPT1 and the organic anion transporter SLCO2B1/OATP2B1 are expressed in intestinal epithelial cells and are involved in uptake transport from the lumen (Hilgendorf et al., 2007; Omkvist et al., 2010; Yoshida et al., 2013). Cells differentiated by using 8-Br-cAMP and IBMX have uptake transporter activities via these transporters (Fig. 5). Furthermore, the activity of PEPT1 was markedly increased by the two compounds (Fig. 5A). 
We have previously reported the method of differentiation into human iPS cell-derived intestinal epithelial cells with transforming growth factor- $\beta$, DNA methyltransferase, and mitogen-activated protein kinase inhibitors and the characteristics of intestinal transport (Iwao et al., 2015; Kodama et al., 2016a,b). However, the cells still did not have sufficient P-gp activity. In the present study, with the use of 8-Br-cAMP and IBMX in addition to the three inhibitors, the TEER values were increased and the human iPS cell-derived intestinal epithelial cells formed more robust tight junctions (Fig. 6, A and B). The cells also had P-gp activity (Fig. 6C). Moreover, the activities of PEPT1 and OATP in Caco-2 cells were 3.7 and $0.07 \mathrm{pmol} / \mathrm{h}$ per milligram protein, respectively (data not shown). In the efflux transport study via P-gp, the apical-to-basal $P_{\text {app }}$ values were $0.49 \pm 0.05$ and $0.56 \pm 0.01$ $\left(\times 10^{-6} \mathrm{~cm} / \mathrm{s}\right)$ in the absence and presence of PSC833, respectively. On the other hand, the basal-to-apical $P_{\text {app }}$ values were $2.73 \pm 0.26$ and $0.63 \pm 0.07\left(\times 10^{-6} \mathrm{~cm} / \mathrm{s}\right)$ in the absence and presence of PSC833, respectively. The ER value, calculated from the apical-to-basal and basal-to-apical $P_{\text {app }}$ values, was decreased from 5.7 to 1.1 by PSC833 (data not shown). Therefore, human iPS cell-derived intestinal cells have almost equivalent functions of uptake and efflux transporters to those of Caco- 2 cells. Based on these results, we believe human iPS cellderived intestinal epithelial cells can serve as a useful tool for the evaluation of PEPT1-, OATP-, P-gp-, and BCRP-mediated transport in the intestinal absorption process.

The activation of cAMP signaling during differentiation of human intestinal epithelial cells from human iPS cells increases the expression levels of intestinal markers and pharmacokinetics-related genes and improves the activities of drug-metabolizing enzymes and drug transporters. Therefore, human iPS cell-derived intestinal epithelial cells differentiated using this novel method can be more useful for predicting the pharmacokinetics in the human small intestine than those differentiated by previously reported methods. This study is the first to prove that activation of cAMP signaling is effective for differentiation into human iPS cell-derived intestinal epithelial cells.

\section{Acknowledgments}

We are grateful to Dr. Hidenori Akutsu, Dr. Yoshitaka Miyagawa, Dr. Hajime Okita, Dr. Nobutaka Kiyokawa, Dr. Masashi Toyoda, and Dr. Akihiro Umezawa for providing human iPS cells (Windy). We also thank Fujifilm Corporation for measuring the metabolites.

\section{Authorship Contributions}

Participated in research design: Kabeya, Iwao, Matsunaga

Conducted experiments: Kabeya, Qiu, Hibino, Nagasaki, Kodama.

Performed data analysis: Kabeya, Qiu, Hibino, Nagasaki, Kodama.

Wrote or contributed to the writing of the manuscript: Kabeya, Iwao, Matsunaga.

\section{References}

Aldhous MC, Shmakov AN, Bode J, and Ghosh S (2001) Characterization of conditions for the primary culture of human small intestinal epithelial cells. Clin Exp Immunol 125:32-40.

Boller K, Arpin M, Pringault E, Mangeat P, and Reggio H (1988) Differential distribution of villin and villin MRNA in mouse intestinal epithelial cells. Differentiation 39:51-57.

Chen L, Wang P, Andrade CF, Zhao IY, Dubé PE, Brubaker PL, Liu M, and Jin T (2005) PKA independent and cell type specific activation of the expression of caudal homeobox gene Cdx-2 by cyclic AMP. FEBS $J$ 272:2746-2759.

Choi MY, Romer AI, Hu M, Lepourcelet M, Mechoor A, Yesilaltay A, Krieger M, Gray PA, and Shivdasani RA (2006) A dynamic expression survey identifies transcription factors relevant in mouse digestive tract development. Development 133:4119-4129.

Chougule P, Herlenius G, Hernandez NM, Patil PB, Xu B, and Sumitran-Holgersson S (2012) Isolation and characterization of human primary enterocytes from small intestine using a novel method. Scand J Gastroenterol 47:1334-1343.

Coskun M, Troelsen JT, and Nielsen OH (2011) The role of CDX2 in intestinal homeostasis and inflammation. Biochim Biophys Acta 1812:283-289.
Darmoul D, Voisin T, Couvineau A, Rouyer-Fessard C, Salomon R, Wang Y, Swallow DM, and Laburthe M (1994) Regional expression of epithelial dipeptidyl peptidase IV in the human intestines. Biochem Biophys Res Commun 203:1224-1229.

Gajda AM and Storch J (2015) Enterocyte fatty acid-binding proteins (FABPs): different functions of liver and intestinal FABPs in the intestine. Prostaglandins Leukot Essent Fatty Acids 93:9-16.

Groschwitz KR and Hogan SP (2009) Intestinal barrier function: molecular regulation and disease pathogenesis. J Allergy Clin Immunol 124:3-20, quiz 21-22.

Hidalgo IJ, Raub TJ, and Borchardt RT (1989) Characterization of the human colon carcinoma cel line (Caco-2) as a model system for intestinal epithelial permeability. Gastroenterology 96 736-749.

Hilgendorf C, Ahlin G, Seithel A, Artursson P, Ungell AL, and Karlsson J (2007) Expression of thirty-six drug transporter genes in human intestine, liver, kidney, and organotypic cell lines. Drug Metab Dispos 35:1333-1340.

Ikuno T, Masumoto H, Yamamizu K, Yoshioka M, Minakata K, Ikeda T, Sakata R, and Yamashita JK (2017) Efficient and robust differentiation of endothelial cells from human induced pluripotent stem cells via lineage control with VEGF and cyclic AMP. PLoS One 12: e0173271.

Iwao T, Kodama N, Kondo Y, Kabeya T, Nakamura K, Horikawa T, Niwa T, Kurose K, and Matsunaga T (2015) Generation of enterocyte-like cells with pharmacokinetic functions from human induced pluripotent stem cells using small-molecule compounds. Drug Metab Dispos 43:603-610.

Iwao T, Toyota M, Miyagawa Y, Okita H, Kiyokawa N, Akutsu H, Umezawa A, Nagata K, and Matsunaga T (2014) Differentiation of human induced pluripotent stem cells into functional enterocyte-like cells using a simple method. Drug Metab Pharmacokinet 29: $44-51$.

Jouan E, Le Vée M, Mayati A, Denizot C, Parmentier Y, and Fardel O (2016) Evaluation of P-glycoprotein inhibitory potential using a Rhodamine 123 accumulation assay. Pharmaceutics 8 (2): 12 .

Kabeya T, Matsumura W, Iwao T, Hosokawa M, and Matsunaga T (2017) Functional analysis of carboxylesterase in human induced pluripotent stem cell-derived enterocytes. Biochem Biophys Res Commun 486:143-148.

Kodama N, Iwao T, Kabeya T, Horikawa T, Niwa T, Kondo Y, Nakamura K, and Matsunaga T (2016a) Inhibition of mitogen-activated protein kinase kinase, DNA methyltransferase, and transforming growth factor- $\beta$ promotes differentiation of human induced pluripotent stem cells into enterocytes. Drug Metab Pharmacokinet 31:193-200.

Kodama N, Iwao T, Katano T, Ohta K, Yuasa H, and Matsunaga T (2016b) Characteristic analysis of intestinal transport in enterocyte-like cells differentiated from human induced pluripotent stem cells. Drug Metab Dispos 44:0.

Landry C, Huet C, Mangeat P, Sahuquet A, Louvard D, and Crine P (1994) Comparative analysis of neutral endopeptidase (NEP) and villin gene expression during mouse embryogenesis and enterocyte maturation. Differentiation 56:55-65.

Lee JS, Paull K, Alvarez M, Hose C, Monks A, Grever M, Fojo AT, and Bates SE (1994) Rhodamine efflux patterns predict P-glycoprotein substrates in the National Cancer Institute drug screen. Mol Pharmacol 46:627-638.

Lennernäs H, Palm K, Fagerholm U, and Artursson P (1996) Comparison between active and passive drug transport in human intestinal epithelial (Caco-2) cells in vitro and human jejunum in vivo. Int $J$ Pharm 127:103-107.

Nakamura T, Sakaeda T, Ohmoto N, Tamura T, Aoyama N, Shirakawa T, Kamigaki T, Nakamura T, Kim KI, Kim SR, et al. (2002) Real-time quantitative polymerase chain reaction for MDR1, MRP1, MRP2, and CYP3A-mRNA levels in Caco-2 cell lines, human duodenal enterocytes, normal colorectal tissues, and colorectal adenocarcinomas. Drug Metab Dispos 30:4-6.

Negoro R, Takayama K, Nagamoto Y, Sakurai F, Tachibana M, and Mizuguchi H (2016) Modeling of drug-mediated CYP3A4 induction by using human iPS cell-derived enterocyte-like cells. Biochem Biophys Res Commun 472:631-636.

Ogaki S, Morooka M, Otera K, and Kume S (2015) A cost-effective system for differentiation of intestinal epithelium from human induced pluripotent stem cells. Sci Rep 5: 17297.

Ogaki S, Shiraki N, Kume K, and Kume S (2013) Wnt and notch signals guide embryonic stem cell differentiation into the intestinal lineages. Stem Cells 31:1086-1096.

Ogawa S, Surapisitchat J, Virtanen C, Ogawa M, Niapour M, Sugamori KS, Wang S, Tamblyn L, Guillemette C, Hoffmann E, et al. (2013) Three-dimensional culture and cAMP signaling promote the maturation of human pluripotent stem cell-derived hepatocytes. Developmen 140:3285-3296.

Omkvist DH, Brodin B, and Nielsen CU (2010) Ibuprofen is a non-competitive inhibitor of the peptide transporter hPEPT1 (SLC15A1): possible interactions between hPEPT1 substrates and ibuprofen. Br J Pharmacol 161:1793-1805.

Ozawa T, Takayama K, Okamoto R, Negoro R, Sakurai F, Tachibana M, Kawabata K, and Mizuguchi H (2015) Generation of enterocyte-like cells from human induced pluripotent stem cells for drug absorption and metabolism studies in human small intestine. Sci Rep 5:16479.

Paine MF, Hart HL, Ludington SS, Haining RL, Rettie AE, and Zeldin DC (2006) The human intestinal cytochrome P450 "pie". Drug Metab Dispos 34:880-886.

Sai Y, Kaneko Y, Ito S, Mitsuoka K, Kato Y, Tamai I, Artursson P, and Tsuji A (2006) Predominant contribution of organic anion transporting polypeptide OATP-B (OATP2B1) to apical uptake of estrone-3-sulfate by human intestinal Caco-2 cells. Drug Metab Dispos 34: $1423-1431$

Sakano D, Shiraki N, Kikawa K, Yamazoe T, Kataoka M, Umeda K, Araki K, Mao D, Matsumoto $\mathrm{S}$, Nakagata N, et al. (2014) VMAT2 identified as a regulator of late-stage $\beta$-cell differentiation. Nat Chem Biol 10:141-148.

Schmiedlin-Ren P, Thummel KE, Fisher JM, Paine MF, Lown KS, and Watkins PB (1997) Expression of enzymatically active CYP3A4 by Caco- 2 cells grown on extracellular matrixcoated permeable supports in the presence of 1alpha,25-dihydroxyvitamin $\mathrm{D}_{3}$. Mol Pharmacol 51:741-754

Spence JR, Mayhew CN, Rankin SA, Kuhar MF, Vallance JE, Tolle K, Hoskins EE, Kalinichenko VV, Wells SI, Zorn AM, et al. (2011) Directed differentiation of human pluripotent stem cells into intestinal tissue in vitro. Nature 470:105-109.

Suh E and Traber PG (1996) An intestine-specific homeobox gene regulates proliferation and differentiation. Mol Cell Biol 16:619-625. 
Sun D, Lennernas H, Welage LS, Barnett JL, Landowski CP, Foster D, Fleisher D, Lee KD, and Amidon GL (2002) Comparison of human duodenum and Caco-2 gene expression profiles for 12,000 gene sequences tags and correlation with permeability of 26 drugs. Pharm Res 19:1400-1416.

Takenaka T, Harada N, Kuze J, Chiba M, Iwao T, and Matsunaga T (2014) Human small intestinal epithelial cells differentiated from adult intestinal stem cells as a novel system for predicting oral drug absorption in humans. Drug Metab Dispos 42:1947-1954.

Wright EM and Turk E (2004) The sodium/glucose cotransport family SLC5. Pflugers Arch 447: $510-518$

Xie F, Ding X, and Zhang QY (2016) An update on the role of intestinal cytochrome P450 enzymes in drug disposition. Acta Pharm Sin B 6:374-383.

Yee S (1997) In vitro permeability across Caco-2 cells (colonic) can predict in vivo (small intestinal) absorption in man—fact or myth. Pharm Res 14:763-766.
Yoshida K, Maeda K, and Sugiyama Y (2013) Hepatic and intestinal drug transporters: prediction of pharmacokinetic effects caused by drug-drug interactions and genetic polymorphisms. Annu Rev Pharmacol Toxicol 53:581-612.

Address correspondence to: Dr. Takahiro Iwao, Department of Clinical Pharmacy, Graduate School of Pharmaceutical Sciences, Nagoya City University, 3-1 Tanabe-dori, Mizuho-ku, Nagoya 467-8603, Japan. E-mail: tiwao@phar. nagoya-cu.ac.jp 